\title{
Optimal Beat Selection Study for QSRT Cancellation Methods in the ECG of Atrial Fibrillation
}

\author{
R Alcaraz ${ }^{1}$, JJ Rieta $^{2}$ \\ ${ }^{1}$ Innovation in Bioengineering Research Group. University of Castilla-La Mancha, Cuenca, Spain \\ ${ }^{2}$ Biomedical Synergy. Valencia University of Technology, Valencia, Spain
}

\begin{abstract}
The proper analysis and characterization of Atrial Fibrillation ( $A F$ ) from surface ECG recordings requires cancelling out ventricular activity (VA), i. e. the QRST complexes. For single-lead ECGs, averaged beat subtraction $(A B S)$ technique is the most widely used. However, this method is very sensitive to QRST wave variations. To date, several strategies have been proposed to overcome this problem, but an exhaustive study about the most suitable set of beats to obtain the most accurate QRST cancellation template, given a concrete recording, has not been performed yet. Thereby, a thorough study about the VA cancellation accuracy, when different complexes are selected to obtain the ventricular template, is presented in this work. The results showed that the most correlationsimilar complexes to the one under cancellation provided a more accurate ventricular cancellation template than the preceding and subsequent beats, thus providing a higher AA extraction quality. In addition, a high number of complexes (greater than 30) is not recommended, because the AA extraction quality improvement is reduced. Nevertheless a more accurate VA representation is obtained when the number of complexes used to generate the QRST cancellation template is increased, but remains below 30 .
\end{abstract}

\section{Introduction}

The surface electrocardiographic (ECG) recording provides a noninvasive way to study atrial fibrillation (AF), which is the most commonly diagnosed cardiac arrhythmia in clinical practice and affects up to $1 \%$ of the general population and $15 \%$ of the population of over 80 years [1]. However, due to the much higher amplitude of the electrical ventricular activity (VA) on the surface ECG, extraction of the atrial activity (AA) is crucial in the study of AF from the ECG. Therefore, the cancellation of the signal components associated with VA, that is, the QRS complex and the T wave, is mandatory [2]. Unfortunately, AA and VA possess overlapped spectral distributions rendering linear filtering solutions unsuccessful [3].

To date, several methods have been proposed to extract the AA signal from surface ECG recordings. The most powerful techniques are those that exploit the spatial diversity of the multilead ECG [4], such as the method that solves the blind source separation problem [3] or the spatiotemporal QRST cancellation strategy [5]. However, the performance of these techniques is seriously reduced when they analyze the early stages of AF, i. e. paroxysmal AF, because the recordings are usually obtained from a Holter system with no more than two or three electrodes, as in this work. In this case, the main alternative is the averaged beat subtraction (ABS) technique, since a reduced number of leads is not sufficient to exploit the spatial information of the ECG. This method relies on the assumption that the average beat can represent each individual beat accurately. However, QRST morphology is often subject to minor changes caused by respiration, patient movement, etc., and, therefore, QRST residua and noise are often present in the remainder ECG [6]. Additionally, in clinical practice the ECG consists of 10 seconds in length recordings and, therefore, a high quality QRST cancellation template may be difficult to obtain [7].

Although different and complex variants have been proposed to overcome this ABS limitations [5, 7], the most suitable complexes selection to obtain the highest quality AA signal has not been studied yet. Thereby, in this work a thorough study about the VA cancellation accuracy, when different complexes are selected to obtain the ventricular cancellation template, is presented for the first time to the scientific community. Thus, some recommendations regarding the optimal number of complexes and their localization into the ECG recording will be indicated.

\section{Materials}

The presented study was validated using a database composed of 25 one minute simulated AF recordings and 50 one minute real ECG signals. Simulated recordings allowed us to compare the estimated and original AA signals, as it was known a priori. Synthesized AF signals 
were created from the combination of AA and VA, which were synthesized separately. The AA was generated from the smooth concatenation of successive TQ segments extracted from real AF recordings. The VA was synthesized from normal sinus rhythm ECG recordings, after P-wave cancellation [8]. In addition, to evaluate the suitability of the algorithm to be applied over real scenarios, which was the final purpose, real AF recordings were used. These signals were obtained from Holter systems of two leads $\left(V_{1}\right.$ and $V_{I I}$ ), digitized at a sampling frequency of $128 \mathrm{~Hz}$ and a resolution of 12 bits. Lead $V_{1}$ was the input signal of the AA estimation approach, as it was the signal with higher AA content [6].

The real ECG recordings were preprocessed in order to reduce noise, nuisance interferences and improve later analysis. Firstly, baseline wander was removed making use of bidirectional high pass filtering with $0.5 \mathrm{~Hz}$ cuttoff frequency [9]. Secondly, high frequency noise was reduced with an eight order bidirectional IIR Chebyshev low pass filtering, whose cut-off frequency was $70 \mathrm{~Hz}$. Finally, powerline interference was removed through adaptive filtering, which preserves the ECG spectral information [10].

\section{Methods}

\subsection{Performance assessment}

The AA estimation performance in simulated recordings was computed by comparing the estimated and the original AA in terms of cross-correlation index $(\rho)$ [8] and mean square error (mse) normalized to the AA root mean square [7]. The cross-correlation index measures the similarities between two signals, and becomes 1 in the case of perfect matching and 0 in the case of completely different and non-related signals:

$$
\rho=\frac{E\left[x_{A A} \widehat{x}_{A A}\right]}{\sigma \widehat{\sigma}}
$$

where $E[\cdot]$ is the expectation operator, and $\sigma$ and $\widehat{\sigma}$ are the standard deviations of $x_{A A}$ and $\widehat{x}_{A A}$, respectively. On the other hand, the normalized mean square error (nmse) is defined as:

$$
n m s e=\sqrt{\frac{\sum_{i=1}^{Q}\left(x_{A A}(i)-\widehat{x}_{A A}(i)\right)^{2}}{\sum_{i=1}^{Q} x_{A A}(i)^{2}}}
$$

being $Q$ the samples of AA involved. This parameter can vary between 0 and 1 , so that high values indicate notable differences between the original and the estimated AA, and values close to zero are associated with very similar AA signals.

The performance of the proposed method was also tested on real ECGs. In these signals, the real AA on the
ECG was obviously unknown. Thereby, a new parameter to estimate the ventricular residua in the extracted AA was proposed. This index considers the area and amplitude of the QRS interval in the AA to evaluate the ventricular residue. Thus, for the $i$-th complex the ventricular residue (VR) was defined as:

$$
\begin{array}{r}
V R_{i}=\frac{1}{\frac{1}{Q} \sum_{i=1}^{Q} \hat{x}_{A A}^{2}} \sqrt{\sum_{k=r_{i}-H}^{r_{i}+H} \widehat{x}_{A A}^{2}} . \\
\cdot \max _{k=r_{i}-H, \ldots, r_{i}+H}\left(\left|\widehat{x}_{A A}\right|\right)
\end{array}
$$

being $2 \cdot H$ the number of samples corresponding to the QRS interval. Note that VR was normalized to the AA root mean square in order to obtain comparable values when signals with different amplitude levels are analyzed. On the other hand, in order to evaluate how the AA extraction algorithm preserves the atrial waveforms in those intervals where the AA should remain unchanged, the similarity (S) parameter was used [11]. This index was evaluated by estimating the cross-correlation coefficient, for each segment free of QRST residua, between the original ECG and the extracted AA.

\subsection{Selection of the most suitable beats}

The AA signal obtained with two different strategies was evaluated using the parameters described in the preceding section. Firstly, a neighbor-based strategy was applied and each QRST complex was cancelled out making use of a ventricular cancellation template generated with the $N / 2$ preceding and $N / 2$ subsequent complexes. If there were not $N / 2$ complexes before the one under cancellation, more subsequent complexes were selected so that the ventricular template were always generated from $N$ beats. In the opposite situation, more preceding beats were selected. Secondly, a correlation-similarity-based strategy was applied and the $N$ most similar QRST complexes to the one under cancellation were used for the template generation. The similarity between QRST complexes was evaluated using the cross-correlation. In both cases, the number of selected complexes, $N$, was varied between 2 and 60.

\section{Results}

Fig. 1 shows mean values for the cross-correlation coefficient, normalized mean square error, ventricular residue and similarity between atrial segments obtained for simulated $\mathrm{AF}$ recordings when the complexes to generate the ventricular cancellation template were selected making use of the two described strategies.

The variations of the ventricular residue and similarity of atrial segments with the number of complexes for the 


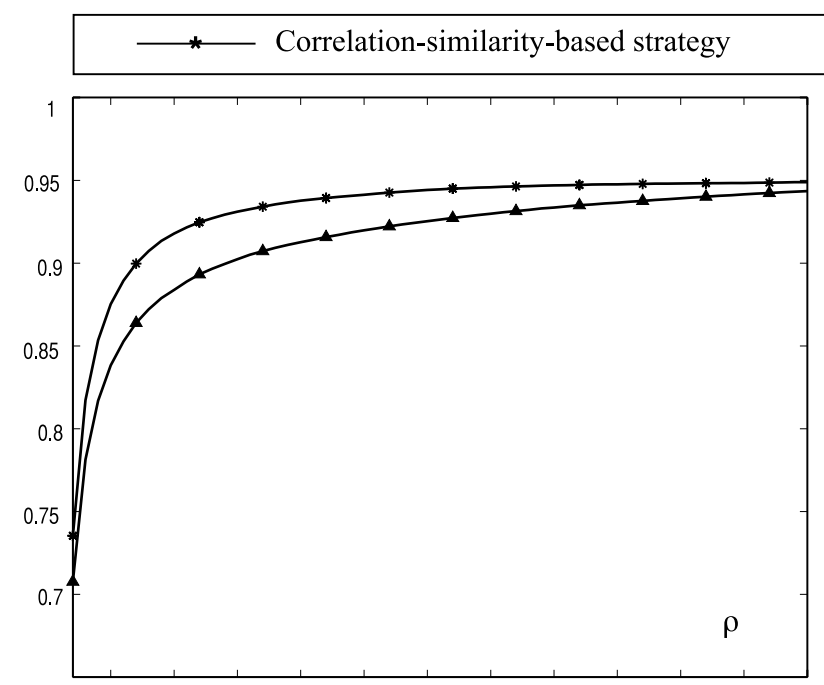

(a)

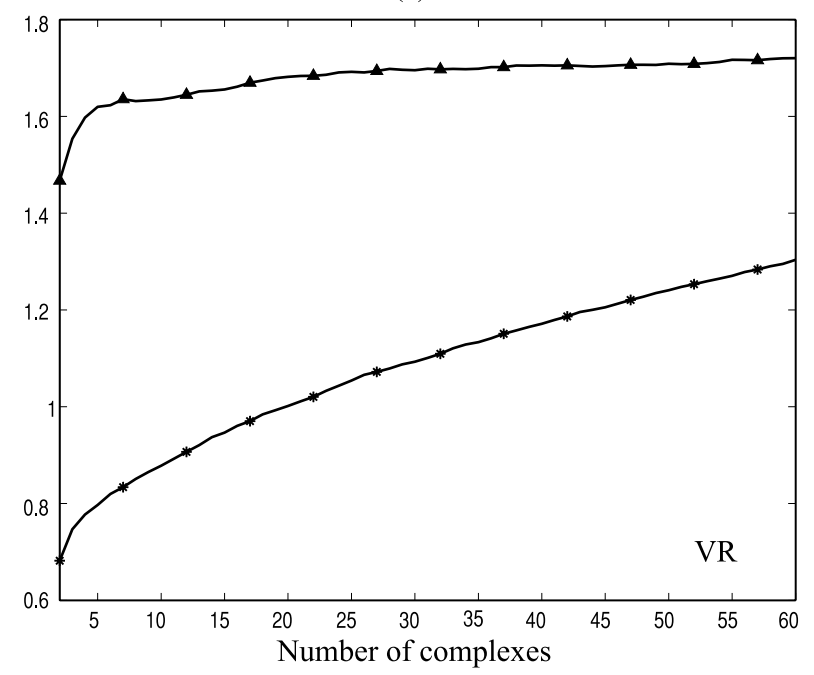

(c)

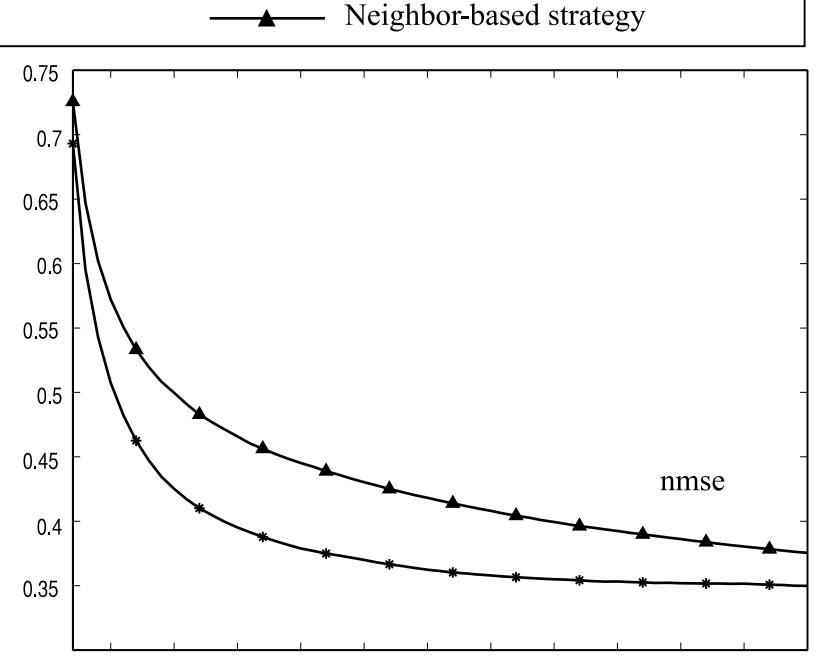

(b)

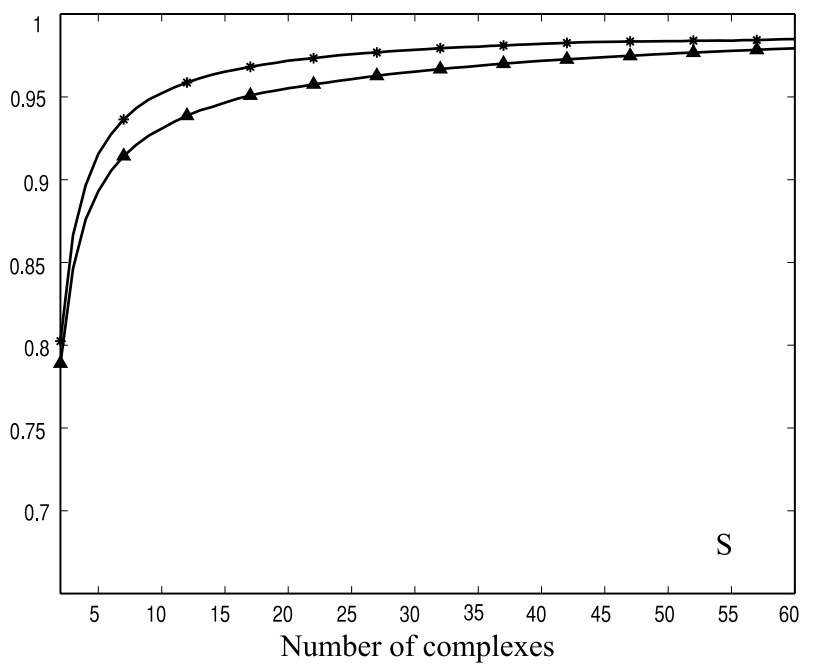

(d)

Figure 1. Results of the optimal complexes selection study obtained for simulated AF recordings. (a) Cross correlation coefficient, (b) normalized mean square error, (c) ventricular residue and (d) similarity of the atrial segments.

real ECGs are presented in Fig. 2. The obtained ventricular residue curves were very similar to those calculated with simulated recordings, presenting similar values. This fact increases the consistency of the defined indices.

\section{Discussion and conclusions}

These obtained performance parameters values allow us to indicate some recommendations useful for any ventricular cancellation method: (1) a higher quality AA extraction is obtained when the most correlation-based similar complexes to the one under cancellation are selected. (2) When short ( $<20$ seconds) single-lead ECG recordings are only available, all the complexes should be used to generated the QRST cancellation template, because higher values of cross-correlation and similarity of atrial segments and a considerably low QRS residue will be obtained. In contrast, (3) when long ( $>20$ seconds) singlelead ECG recordings are available, the selection of a number of complexes between 20 and 30 is recommended because a higher number causes a considerably large QRS residue and a lower number obtains a poorer AA extraction in terms of the cross-correlation index and the similarity of atrial segments.

\section{Acknowledgements}

This work was partly supported by the projects PAID04-08 and TEC2007-64884 from the Spanish Ministry of Education and Science. 


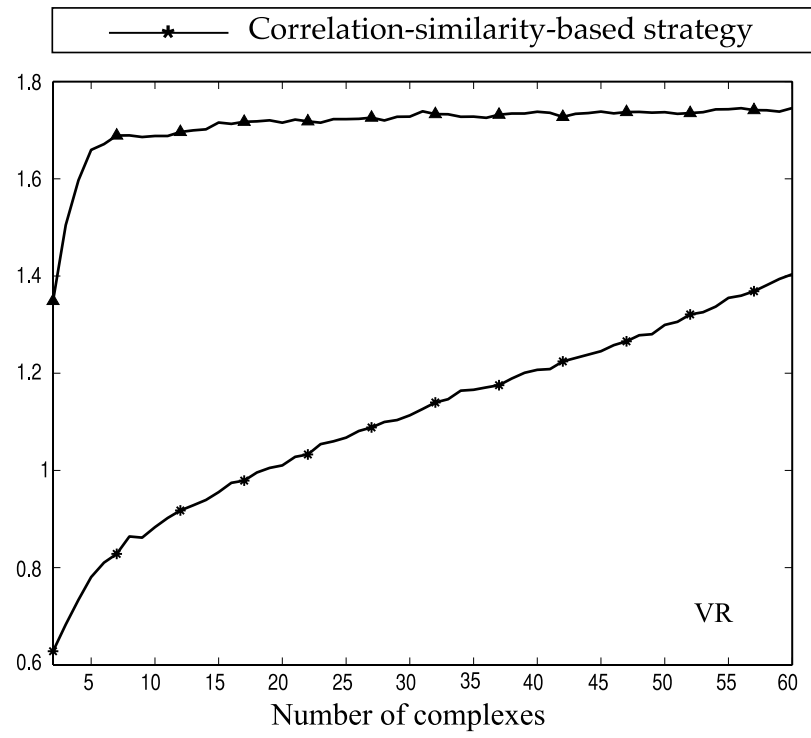

(a)

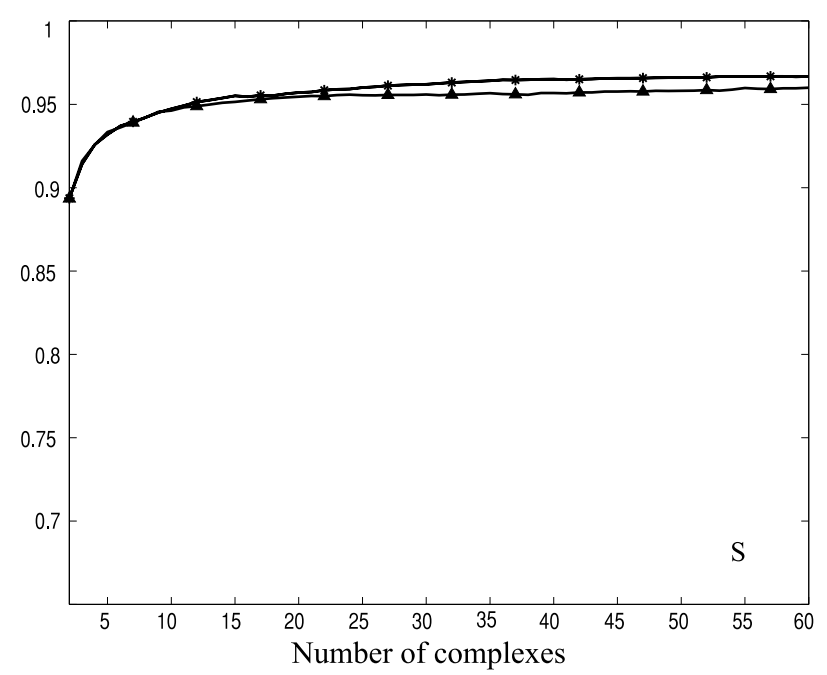

(b)

Figure 2. Results of the optimal complexes selection study obtained for real ECGs. (a) ventricular residue and (b) similarity of the atrial segments.

\section{References}

[1] Kannel WB, Wolf PA, Benjamin EJ, Levy D. Prevalence, incidence, prognosis, and predisposing conditions for atrial fibrillation: population-based estimates. Am J Cardiol Oct 1998;82(8A):2N-9N.

[2] Slocum J, Sahakian A, Swiryn S. Diagnosis of atrial fibrillation from surface electrocardiograms based on computerdetected atrial activity. J Electrocardiol Jan 1992;25(1):1-8.

[3] Rieta JJ, Castells F, Sánchez C, Zarzoso V, Millet J. Atrial activity extraction for atrial fibrillation analysis using blind source separation. IEEE Trans Biomed Eng Jul 2004; 51(7):1176-1186.

[4] Langley P, Rieta JJ, Stridh M, Millet J, Sörnmo L, Murray A. Comparison of atrial signal extraction algorithms in 12lead ECGs with atrial fibrillation. IEEE Trans Biomed Eng Feb 2006;53(2):343-346.

[5] Stridh M, Sörnmo L. Spatiotemporal QRST cancellation techniques for analysis of atrial fibrillation. IEEE Trans Biomed Eng Jan 2001;48(1):105-111.

[6] Petrutiu S, Ng J, Nijm GM, Al-Angari H, Swiryn S, Sahakian AV. Atrial fibrillation and waveform characterization. A time domain perspective in the surface ECG. IEEE Eng Med Biol Mag 2006;25(6):24-30.

[7] Lemay M, Vesin JM, van Oosterom A, Jacquemet V, Kap- penberger L. Cancellation of ventricular activity in the ECG: Evaluation of novel and existing methods. IEEE Trans Biomed Eng Mar 2007;54(3):542-546.

[8] Castells F, Mora C, Rieta JJ, Moratal-Pérez D, Millet J. Estimation of atrial fibrillatory wave from single-lead atrial fibrillation electrocardiograms using principal component analysis concepts. Med Biol Eng Comput Sep 2005; 43(5):557-560.

[9] Dotsinsky I, Stoyanov T. Optimization of bi-directional digital filtering for drift suppression in electrocardiogram signals. J Med Eng Technol 2004;28(4):178-180.

[10] Ferdjallah M, Barr RE. Adaptive digital notch filter design on the unit circle for the removal of powerline noise from biomedical signals. IEEE Trans Biomed Eng Jun 1994; 41(6):529-536.

[11] Rieta JJ, Hornero F. Comparative study of methods for ventricular activity cancellation in atrial electrograms of atrial fibrillation. Physiol Meas 2007;28(8):925-936.

Address for correspondence:

Raúl Alcaraz Martínez

E. U. Politécnica de Cuenca

Campus Universitario

16071 Cuenca (Spain)

raul.alcaraz@uclm.es 